\title{
Macropinocytosis: A Metabolic Adaptation to Nutrient Stress in Cancer
}

\author{
Maria Victoria Recouvreux and Cosimo Commisso* \\ Tumor Initiation and Maintenance Program, NCl-Designated Cancer Center, Sanford Burnham Prebys Medical Discovery \\ Institute, La Jolla, CA, United States
}

Oncogenic mutations, such as Ras mutations, drive not only enhanced proliferation but also the metabolic adaptations that confer to cancer cells the ability to sustain cell growth in a harsh tumor microenvironment. These adaptations might represent metabolic vulnerabilities that can be exploited to develop novel and more efficient cancer therapies. Macropinocytosis is an evolutionarily conserved endocytic pathway that permits the internalization of extracellular fluid via large endocytic vesicles known

OPEN ACCESS

Edited by:

Albert Giralt,

University of Lausanne,

Switzerland

Reviewed by:

Mara Sherman,

Oregon Health \&

Science University,

United States

Brian Yee Hong Lam,

University of Cambridge,

United Kingdom

*Correspondence:

Cosimo Commisso

ccommisso@sbpdiscovery.org

Specialty section:

This article was submitted

to Cellular Endocrinology,

a section of the journal

Frontiers in Endocrinology

Received: 22 July 2017 Accepted: 20 September 2017 Published: 29 September 2017

Citation:

Recouvreux MV and Commisso C (2017) Macropinocytosis: A Metabolic Adaptation to Nutrient Stress in Cancer.

Front. Endocrinol. 8:261. doi: 10.3389/fendo.2017.00261 as macropinosomes. Recently, macropinocytosis has been determined to function as a nutrient-scavenging pathway in Ras-driven cancer cells. Macropinocytic uptake of extracellular proteins, and their further degradation within endolysosomes, provides the much-needed amino acids that fuel cancer cell metabolism and tumor growth. Here, we review the molecular mechanisms that govern the process of macropinocytosis, as well as discuss recent work that provides evidence of the important role of macropinocytosis as a nutrient supply pathway in cancer cells.

Keywords: macropinocytosis, Ras, growth factors, nutrient uptake, cancer metabolism

\section{INTRODUCTION}

Sustained rapid proliferation represents a major metabolic hurdle for cancer cells. The challenge lies in balancing the towering energy and nutrient demands required for biomass production with the harsh nutrient-depleted conditions of the tumor microenvironment. Hence, it is not surprising that tumors have evolved the capacity to employ the same oncogenic signaling pathways [e.g., RAS, MYC, PI3-kinase (PI3K)] that trigger aberrant growth to also control the metabolic rewiring that is necessary to adapt to a nutrient-deprived ecosystem (1).

Metabolic reprogramming is now recognized as one of the hallmarks of cancer cells (2) and the topic has become of increasing interest in recent years. Importantly, a better understanding of the molecular mechanisms and metabolic adaptations that confer growth and survival advantages to cancer cells could lead to the discovery of novel therapeutic opportunities. One of the many adaptive strategies that cancer cells use to fulfill their metabolic demands is the ability to exploit alternative nutrient acquisition pathways (3). Among them, macropinocytosis is an evolutionarily conserved form of bulk endocytosis by which cells incorporate extracellular fluid into large, irregularly shaped vesicles called macropinosomes (4). Macropinocytosis was first observed microscopically in malignant cells in the 1930s (5), and since then, it has been extensively studied in different cell types and in varying contexts. For example, the amoeboid organism Dictyostelium discoideum utilizes macropinocytic uptake to engulf fluid and nutrients during axenic growth (6). In antigen presentation 
that occurs in the mammalian immune system, macrophages and dendritic cells employ macropinocytosis to internalize and process extracellular antigenic proteins (7). Macropinocytosis can occur at basal rates, as it occurs spontaneously in many cells, or it can be dramatically induced by receptor tyrosine kinase (RTK) activation or by oncogenes such as Ras (8) and $\mathrm{v}$-Src (9). Although having been observed in transformed cells for nearly 30 years, the biological relevance of macropinocytosis in cancer has only recently been elucidated (10). In cancer cells harboring oncogenic Ras mutations, macropinocytosis serves as a nutrient uptake pathway by which extracellular protein is internalized and degraded to supply the much-needed amino acids that support cellular growth (10-12). This novel function of macropinocytosis as a feeding mechanism in tumors has provided a new perspective for cancer metabolism research and has positioned this ancient mechanism as a promising target for therapeutic intervention.

In this review, we aim to summarize the mechanisms that govern macropinocytosis regulation in cancer cells, as well as to provide the latest findings supporting its important role as a nutrient supply pathway that enables tumor cell proliferation and survival.

\section{REGULATION OF MACROPINOCYTOSIS IN CANCER CELLS}

Macropinocytosis is a clathrin-independent endocytic process driven by actin. In contrast to the closely related phagocytosis pathway, macropinocytic uptake is non-selective and not controlled by its cargo (13). Other distinctive features that define macropinocytosis are the fact that it can be stimulated by growth factors, and that it is suppressed by ion exchange inhibitors such as amiloride and its derivatives, which specifically inhibit macropinocytosis as opposed to other endocytic pathways $(4,14)$. Another definitive property is that macropinosomes are larger than other endocytic vesicles and can be specifically labeled by high molecular weight dextrans (15), which are incorporated into discrete vesicles larger than $0.2 \mu \mathrm{m}$ in diameter.

Macropinocytosis is intimately linked to actin cytoskeleton dynamics. Protrusions of the plasma membrane, known as membrane ruffles, are formed via actin polymerization. Nascent macropinosomes arise at the cell surface of ruffling cells when these membrane protrusions spontaneously form cup-shaped ruffles that close, leading to fission of the nascent macropinosome from the plasma membrane and the internalization of extracellular fluid (13). Two types of membrane ruffles have been described and both can lead to macropinocytosis: planar ruffles, which are derived from the cell edges, and circular dorsal ruffles, which occur at the apical cell surface (16).

Both membrane ruffling and macropinocytosis depend heavily on actin cytoskeleton remodeling, as evidenced by their complete abrogation by inhibitors that disrupt actin polymerization, like cytochalasin $\mathrm{D}(17,18)$. Moreover, many key regulators of actin polymerization, such as members of the Ras superfamily of small guanosine triphosphatases (GTPases), Ras, Rac, Cdc42, Arf6, and Rab5, among others, have been associated with ruffle formation and macropinocytic activity (19). Additionally, membrane phospholipids, in particular phosphatidylinositol (PI), PI4P, PI5P, PI $(4,5) \mathrm{P}_{2}$, and $\mathrm{PI}(3,4,5)$ $\mathrm{P}_{3}$, and the phospholipid kinases and phosphatases that interconvert them, are important players in the spatiotemporal regulation of macropinocytosis (13). For instance, inhibition of PI3K by either wortmannin or LY294002 has been shown to abolish macropinocytosis in several cell types including cancer cells, fibroblasts, and macrophages (18, 20, 21). Stage-specific enrichment of each type of PI during macropinosome maturation allows for the sequential recruitment and activation of specific enzymes and adapter proteins, including small GTPases and other proteins necessary for actin polymerization and membrane trafficking, such as Scar/Wave, Wasp, and Arp2/3 complexes, as well as sorting nexins (22-24).

These orchestrated rearrangements of the actin cytoskeleton, as well as the production and turnover of phospholipids necessary for macropinocytosis, can be initiated by growth factor-dependent activation of RTKs (Figure 1). Induction of macropinocytosis by growth factors, such as epidermal growth factor (EGF), platelet-derived growth factor (PDGF), and macrophage colony-stimulating factor, has been well studied and relies on the capacity of growth factors to stimulate membrane ruffling through the activation of the small GTPases Ras and $\operatorname{Rac}(14,25-27)$. Several studies have demonstrated that Ras activation, either by growth factor stimulation or through oncogenic mutation, leads to increased membrane ruffling and macropinocytosis. First observations by Bar-Sagi and Feramisco showed that microinjection of oncogenic Hras into rat embryo fibroblasts rapidly induced ruffles and fluid-phase uptake of high molecular weight dextran (8). Similarly, Kras-transformed Rat-1 fibroblasts showed increased macropinocytosis that was dependent on PI3K and phospholipase C activity (18). More recently, it was shown that human bladder and pancreatic cancer cells that harbor oncogenic HRAS or KRAS mutations, respectively, display an enhancement of macropinocytosis relative to cancer cells of the same tissue type that express wild-type HRAS or KRAS (10). Furthermore, macropinocytic activity was also observed in vivo in a Kras-mutant mouse model of pancreatic ductal adenocarcinoma (PDAC) $(10,28)$, as well as in human primary PDAC specimens (11).

Ras activation leads to the stimulation of a plethora of different signal transduction pathways, including Rac, Cdc42, PI3K, and Raf/Erk activation. Activation of Rac by Ras has been shown to have an important role in inducing both membrane ruffling and macropinocytosis in different cell types (26). Both Rac1 transient activation and subsequent deactivation are required for complete closure and maturation of macropinosomes (29). Interestingly, Rac1 and Cdc42 are necessary and sufficient to induce macropinocytosis uptake in bladder cancer cells, as demonstrated by employing dominant negative and constitutively active forms of these small GTPases (30). In addition, it was recently demonstrated that Dock1, a Rac-specific guanine nucleotide exchange factor, is required for oncogenic Rasinduced macropinocytosis in several cancer cells (31).

$\mathrm{Rac}$ and $\mathrm{Cdc} 42$ can activate actin polymerization via p21-activated kinase 1 (Pak1), which has been shown to 


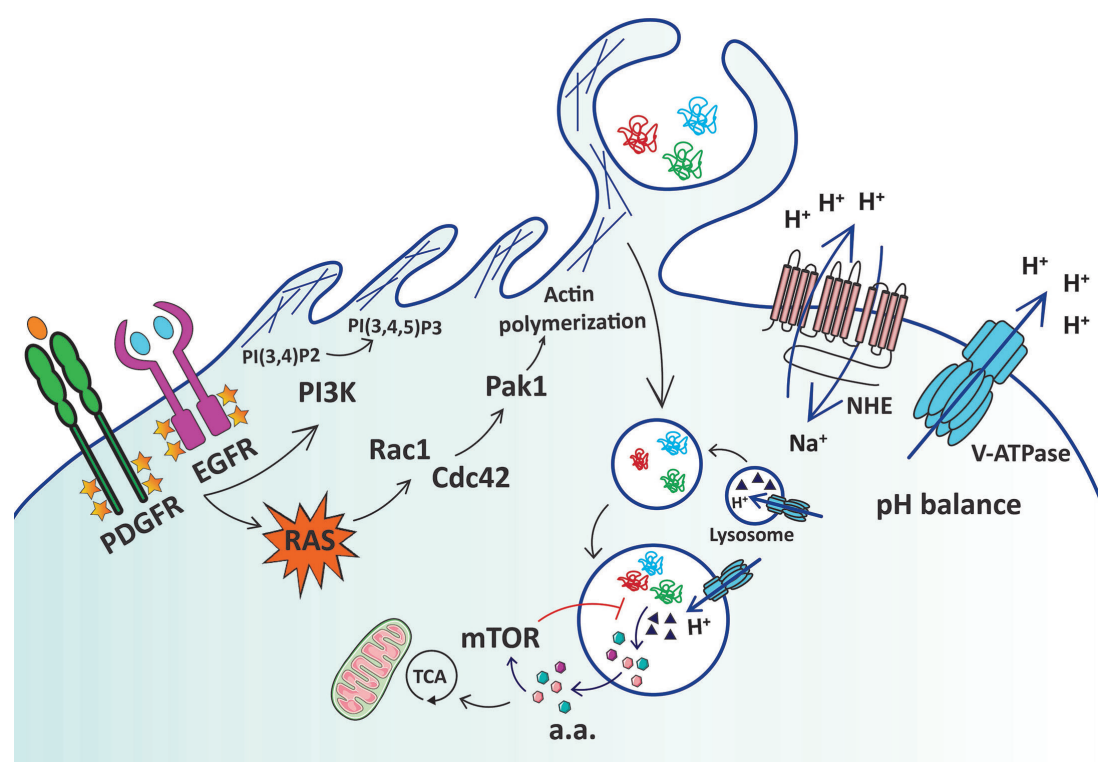

FIGURE 1 | Schematic representation of extracellular protein uptake via macropinocytosis in cancer cells. Ras activation, either by growth factor stimulation or through oncogenic mutation, leads to increased membrane ruffling and macropinocytosis via activation of Rac1 and Cdc42, which in turn stimulate p21-activated kinase 1 (Pak1) to induce actin polymerization. Activation of Rac1 and $\mathrm{Cdc} 42$ is sensitive to changes in submembranous $\mathrm{pH}$, and the activity of $\mathrm{Na}+/ \mathrm{H}+\mathrm{exchangers}$ (NHEs) and vacuolar H+-ATPase (V-ATPases) is crucial to maintaining pH homeostasis. Conversion of membrane phosphoinositides by PI3-kinase (PI3K) is also necessary for macropinocytosis. Macropinosomes containing extracellular proteins such as albumin and collagen are internalized and subsequently fuse with lysosomes. Lysosomal proteases ( $\mathbf{A}$ ) allow the catabolism of extracellular proteins into free amino acids (a.a.) that can fuel the TCA cycle to promote cell growth and survival. mTORC1 finely regulates the utilization of extracellular protein-derived amino acids by inhibiting macropinocytosed protein catabolism when free a.a. are abundant. Yellow stars represent phosphorylation of growth factor receptors.

co-localize with macropinosomes in $3 \mathrm{~T} 3$ fibroblasts, and can drive macropinocytosis through circular dorsal ruffles when expressed in a constitutively active form $(32,33)$. Pak1 activity is also important for closure of macropinocytic cups through phosphorylation of CtBP1/BARS, a protein involved in membrane fission in the context of EGF-induced macropinocytosis (34). Signaling through other members of the Rab family of small GTPases, such as Rab5 and its effector Rabankyrin-5, have been shown to play a role in macropinosome formation and maturation. Specifically, both have been shown to be associated with membrane ruffles in epithelial cells $(35,36)$ and expression of a Rab5 dominant negative form inhibits PDGF-stimulated circular ruffles in MEFs (37).

While Ras, Rac, and Cdc42 signaling are critical for the early steps of macropinocytosis (i.e., membrane ruffling and formation of macropinocytic cups), PI3K activity seems to be specifically required for macropinosome closure in tumor cells and macrophages $(18,20,21)$. Studies in the epithelial carcinoid cell line A431 have shown that PI3K inhibitors do not affect EGFRinduced membrane ruffling, but they inhibit macropinocytosis (21). These studies showed that as the macropinocytic cups close, $\mathrm{PI}(3,4) \mathrm{P} 2$ is depleted from the cup while $\mathrm{PI}(3,4,5) \mathrm{P} 3$ production by $\mathrm{PI} 3 \mathrm{~K}$ increases, and these highly regulated kinetics may be required for coordinated actin remodeling that allows macropinosome closure. It should be noted that these observations may be cell and/or stimuli specific, as other studies have demonstrated that PI3K can control plasma membrane ruffling caused by either oncogenic v-Src expression or PDGF stimulation $(18,38)$. The regulation of membrane ruffling by $\mathrm{PI} 3 \mathrm{~K}$ in these contexts may be mediated by PI3K-dependent activation of Rac1 (39).

The fate of macropinosomes after internalization varies depending on the cell type, as they can be recycled to the cell membrane as is the case in A431 cells (40) or they can adopt degradative properties by fusing with lysosomes and undergoing a lysosome-dependent acidification, as is the case in macrophages (41) and Ras-transformed cancer cells (10). Although the mechanisms underlying macropinosome maturation remain to be explored, a switch from Rab5 to Rab7 accumulation on the macropinosome (42) and the recruitment of specific septins to the maturing macropinosome, seem to regulate fusion events with late endosomal/lysosomal compartments (43). Although much work has been done in past years to elucidate the mechanisms that control macropinocytosis, more studies are necessary to further identify the cell- and tissue-specific pathways that regulate this process, especially in cancer cells where it can represent a therapeutic targeting strategy.

\section{MACROPINOCYTOSIS AND pH HOMEOSTASIS}

As discussed above, macropinocytic induction arises as a result of the coordinated interactions among small GTPases, actin filaments, and membrane phosphoinositides in restricted areas of the plasma membrane known as membrane ruffles. Because 
of the electrostatic origin of some of these interactions, they are susceptible to alterations in the charge balance across the plasma membrane. Accordingly, quite soon after the discovery of growth factor-induced macropinocytosis, it was shown that conditions that acidify the cytosol, such as the addition of $\mathrm{NH}_{4}^{+}$, or the blockade of the $\mathrm{Na}^{+} / \mathrm{H}^{+}$exchangers (NHEs) by amiloride, dramatically inhibit macropinocytosis (14). Given that NHE inhibition selectively blocks macropinocytosis, leaving coated vesicles intact, sensitivity to amiloride and its analogs, like 5-( $N$-ethyl- $N$-isopropyl) amiloride (EIPA) and HOE-694, has been used as a distinctive feature of macropinocytosis (4). Nevertheless, the functional association between $\mathrm{Na}^{+} / \mathrm{H}^{+}$ exchange and macropinocytosis and the mechanisms mediating amiloride/EIPA inhibition remained unknown for many years.

Koivusalo et al. described that targeting of NHEs, by amiloride and HOE-694, abrogates EGF-induced macropinocytosis by lowering the submembranous $\mathrm{pH}$ (44). Interestingly, these changes in $\mathrm{pH}$ (from $\mathrm{pH}=7.8$ to 6.8 ) seemed to exclusively affect the recruitment and activation of Racl and Cdc42 to membrane ruffles. Consequently, the recruitment of their downstream effectors, Pak1 and Arp2/3, was also abrogated, thus inhibiting ruffle formation without affecting EGFR phosphorylation or PI3K activation. The authors also showed that EGF could stimulate $\mathrm{Na}^{+} / \mathrm{H}^{+}$exchange with a concomitant alkalinization of the cytoplasm at sites of nascent macropinosome formation. Inhibition of the NHEs resulted in an accumulation of acidic equivalents, which are thought to occur due to a boost of metabolic activity driven by EGF stimulation. The outcome was an overall acidification of the cytoplasm, with a more pronounced effect in the vicinity of the plasma membrane where macropinocytosis was taking place (44). Although this study only addressed NHE inhibition of EGF-induced macropinocytosis in A431 cells, it is likely that similar mechanisms account for amiloride/ EIPA inhibition in the setting of other growth factors and in Ras-induced macropinocytosis, where $\mathrm{H}^{+}$accumulation caused by increased metabolic activity and actin polymerization also occurs, especially in cancer cells where the high metabolic rate is well known to promote acidification of the cell and the tumor microenvironment (45-47).

The most commonly used macropinocytosis inhibitors, amiloride and EIPA, broadly target the SLC9A gene family of NHEs, which includes 11 isoforms reported to date (48). Whether specific NHE isoforms differentially regulate macropinocytosis remains an open question. Although NHE1 is highly expressed in several cancer cell lines (49) and is the most widely studied isoform, gene expression analyses indicate that NHE6, 7, and 8 are expressed at levels comparable to NHE1 in PDAC cells (50); therefore, it would be useful to conduct further studies aimed at identifying the contribution of specific NHE isoforms to macropinocytosis.

Underscoring the importance of NHE-dependent macropinocytosis in tumor growth, EIPA treatment of mice bearing MIA-PaCa2-derived xenograft tumors showed a suppression of intratumoral macropinocytosis that was accompanied by a reduction in tumor size relative to control mice (10). Moreover, EIPA treatment was effective only in tumors with high macropinocytic activity, as tumor growth rate was not affected in tumors derived from BxPC3 cells, which display low levels of macropinocytosis. These results indicated that enhanced macropinocytic activity in particular tumors might represent a metabolic vulnerability that can be specifically harnessed to restrain tumor growth.

In addition to nascent macropinosome formation, later stages of macropinosome maturation where the degradation of the macropinocytosed cargo occurs are also dependent on $\mathrm{pH}$ regulation. Macropinosome maturation includes fusion with lysosomes, which facilitates the delivery of the machinery necessary for compartmental acidification and the lysosomal proteases that are responsible for protein catabolism (41). Vacuolar $\mathrm{H}^{+}$-ATPases (V-ATPases) function to maintain the acidic $\mathrm{pH}$ of different intracellular organelles such as late endosomes and lysosomes and these proton pumps play a critical role in vesicular trafficking and protein degradation (51). Concordantly, inhibition of V-ATPases by bafilomycin A1 has been shown to impair downstream events in the macropinocytosis pathway (Figure 1). For instance, bafilomycin A1 inhibits degradation of LDL causing its accumulation in macropinocytic vesicles in macrophages (52). Furthermore, protein degradation of macropinocytosed albumin was prevented by treatment with bafilomycin A1 in KRAS-mutant PDAC cells (10). Interestingly, bafilomycin A 1 also inhibits the initial stages of macropinocytosis in HRAS-mutant T24 bladder cells (53), as well as in KRAS-mutant A549 lung cells (54). These effects of bafilomycin on nascent macropinosome formation may be a result of either (1) perturbations of cytosolic $\mathrm{pH}$ due to impaired pumping of protons into lysosomes or (2) alterations of submembranous $\mathrm{pH}$ due to inhibition of proton pumping to the extracellular space. Supporting the second possibility, several studies have demonstrated that V-ATPases can reside at the plasma membrane in KRAS-mutant PDAC and breast tumor cells and contribute to $\mathrm{pH}$ regulation at the plasma membrane $(55,56)$.

Altogether, these results suggest that $\mathrm{pH}$ homeostasis, both at the cellular and organellular level, is vital to properly execute the macropinocytosis program. Therefore, from the perspective of the pathological state, $\mathrm{pH}$ homeostasis is at the center of cancer cell metabolism and is critical to the role of macropinocytosis as a vital nutrient supply route that supports cancer cell growth and survival.

\section{MACROPINOCYTOSIS AS A SURVIVAL STRATEGY IN TUMORS}

Oncogenic Ras triggers a myriad of cellular adaptations to promote the metabolic rewiring that allows cancer cells to sustain unrestrained proliferation [reviewed in Ref. (57)]. Such rewiring includes enhanced glucose uptake and glycolytic activity, shifts in glutamine metabolism and redox balance $(58,59)$, increased flux of glucose to anabolic pathways such as hexosamines and ribose-5-phosphate (60), as well as upregulation of the major nutrient-scavenging mechanisms: autophagy (61-63) and macropinocytosis (10). It was recently demonstrated that extracellular amino acids and lipids rather than glucose contribute to the majority of cell biomass in proliferating cancer cells (64). These findings underscore the relevance of nutrient-scavenging 
pathway exploitation by cancer cells to support tumor growth and survival.

Unlike autophagy, which generates nutrients from a limited supply of intracellular organelles and cytosolic proteins, macropinocytosis serves as a feeding mechanism by internalizing and degrading extracellular proteins. In this way, the resulting protein-derived amino acids can be utilized by the tumor to fuel central carbon metabolism, in addition to other metabolic pathways. This function has been proven to be particularly relevant to sustaining tumor growth in nutrientdeprived environments both in vitro and in vivo. For instance, mutant KRAS-driven pancreatic cancer cells, which rely on glutamine metabolism to support their growth, can maintain proliferation under glutamine-limiting conditions if supplied with extracellular serum albumin. Importantly, this escape from the deleterious effects of glutamine deprivation is suppressed by the inhibition of macropinocytosis (10). Similarly, extracellular serum albumin has the ability to reverse a proliferation arrest in PDAC cells grown in the absence of essential amino acids, such as leucine $(11,12)$. Tracing experiments with labeled extracellular protein showed that protein-derived amino acids are indeed incorporated into TCA cycle metabolites, supporting the growth-promoting role of macropinocytosis in Ras-transformed cells (10). Thus, macropinocytosis is necessary to support cell growth under nutrient-deprived conditions. Whether the extent of macropinocytosis can be dialed up or down depending on nutritional status remains to be elucidated.

The potential importance of macropinocytosis in human cancer is underscored by the observation that PDAC tumor tissues from Whipple procedure patients display enhanced macropinocytic uptake relative to adjacent non-neoplastic regions (11). Such PDAC tumors are hypovascularized and are depleted of amino acids (11); therefore, extracellular protein scavenging represents an attractive alternative for nutrient acquisition in these tumors as opposed to the import of free, circulating amino acids, which would depend on adequate perfusion. In agreement with this, extracellular protein uptake and catabolism via macropinocytosis has been directly evidenced in vivo in murine PDAC tumors (28). Moreover, inhibition of macropinocytosis via EIPA treatment suppressed tumor growth in xenograft tumors (10). Although the majority of the studies on the utilization of extracellular protein as a nutrient source have been performed in pancreatic cancer cells and animal models, similar results have also been reported in Ras-driven cancer cells of different tissue origins, such as bladder, lung, sarcoma, and colon cancer (10, $31,65)$. The cell growth effects mediated by macropinocytosis under glutamine-deprived conditions were suppressed in KRASmutant lung, sarcoma, and colon cells, when the Racl activator Dock1 was inhibited either pharmacologically or by genetic ablation. Moreover, treatment with a Dock1 inhibitor decreased tumor growth and metastasis in mice (31). In addition to tumor cells, macropinocytosis was also observed in the stromal compartment of PDAC tumors (11). Further studies are necessary to evaluate the potential contribution of macropinocytosis to survival and growth of these cells.

The intracellular degradation of extracellular proteins acquired via macropinocytosis is dependent upon lysosomes, which are tightly controlled by the mammalian target of rapamycin complex (mTORC1), a key regulator of cell growth that responds to nutrient availability (66). Lysosomal catabolism of extracellular proteins has been shown to activate mTORC1 in Ras-transformed cells (12), and furthermore, macropinocytosisderived lysosomal amino acids are required for rapid activation of mTORC1 in response to growth factor stimulation to promote cell growth (67). On the other hand, mTORC1 activation seems to minimize lysosomal degradation of macropinocytosed proteins. Suppression of mTORC1 by rapamycin or torin 1 enhances protein catabolism and proliferation in amino acid-starved PDAC cells in vitro, and in hypovascularized, nutrient-poor regions of PDAC tumors (12). Thus, although it seems that mTORC1 does not directly regulate the early steps of macropinocytosis, it does play a pivotal role in regulating the degradation of proteins that are internalized by macropinosomes, allowing for the coordination of protein catabolism in response to nutrient availability. Hence, when free amino acids are plentiful, mTORC1 activation could suppress the catabolism of macropinocytosed proteins and conversely, as amino acid levels decrease upon consumption, mTORC1 suppression could allow for enhanced protein degradation. It is also conceivable that as protein-derived amino acids are produced, they, in turn, activate mTORC1, establishing a feedback regulatory loop that serves to shift between nutrient acquisition pathways depending on nutrient availability (Figure 1).

Finally, molecules other than serum albumin are also internalized via macropinocytosis, and uptake of these molecules might also contribute to tumor metabolism and growth. For example, tumor cells can internalize and catabolize extracellular matrix molecules, such as fibronectin and collagen $(28,68)$. Like serum albumin, internalization of these matrix molecules serves to produce protein-derived amino acids that can support tumor cell growth. In addition to extracellular proteins, KRAS-mutant A549 lung cancer cells can also macropinocytose extracellular ATP in order to increase the intracellular ATP pool (69). Ras-driven cancer cells are also known to have increased lipid scavenging to support tumor metabolism $(70,71)$, and although the mechanisms remain to be elucidated, it is reasonable to hypothesize that serum lipids bound to albumin are taken up via macropinocytosis.

\section{CONCLUDING REMARKS}

The critical role that metabolic reprogramming and adaptation strategies play in supporting the growth of tumors is now widely recognized. By inducing the internalization of extracellular proteins, and other macromolecules, that can be further processed and utilized to fuel different metabolic pathways, macropinocytosis provides not only a survival mechanism under nutrientscarce conditions but also the potential for unrestricted tumor growth in an adverse tumor microenvironment. For this reason, targeting macropinocytosis has emerged as a novel therapeutic strategy that requires further investigation. Understanding the molecular events that drive macropinocytosis in the context of different cancers might inform the design of more specific and potent inhibitors. Given that macropinocytosis is crucial to 
sustaining tumor growth under nutrient-deprived conditions, it is possible that macropinocytosis inhibition would be particularly beneficial in patients suffering from severely hypoxic or hypovascularized tumors, such as PDAC. Moreover, studies focused on combination therapies employing macropinocytosis inhibitors in conjunction with other metabolic pathway inhibitors could pave the way for improved therapeutic outcomes.

\section{AUTHOR CONTRIBUTIONS}

MR and CC conceived, organized, and wrote the manuscript.

\section{REFERENCES}

1. Deberardinis RJ, Sayed N, Ditsworth D, Thompson CB. Brick by brick: metabolism and tumor cell growth. Curr Opin Genet Dev (2008) 18(1):54-61. doi:10.1016/j.gde.2008.02.003

2. Hanahan D, Weinberg RA. Hallmarks of cancer: the next generation. Cell (2011) 144(5):646-74. doi:10.1016/j.cell.2011.02.013

3. Pavlova NN, Thompson CB. The emerging hallmarks of cancer metabolism. Cell Metab (2016) 23(1):27-47. doi:10.1016/j.cmet.2015.12.006

4. Kerr MC, Teasdale RD. Defining macropinocytosis. Traffic (2009) 10(4): 364-71. doi:10.1111/j.1600-0854.2009.00878.x

5. Lewis WH. Pinocytosis by malignant cells. Am J Cancer (1937) 29(4):666-79. doi:10.1158/ajc.1937.666

6. Hacker U, Albrecht R, Maniak M. Fluid-phase uptake by macropinocytosis in Dictyostelium. J Cell Sci (1997) 110(Pt 2):105-12.

7. Sallusto F, Cella M, Danieli C, Lanzavecchia A. Dendritic cells use macropinocytosis and the mannose receptor to concentrate macromolecules in the major histocompatibility complex class II compartment: downregulation by cytokines and bacterial products. J Exp Med (1995) 182(2):389-400. doi:10.1084/jem.182.2.389

8. Bar-Sagi D, Feramisco JR. Induction of membrane ruffling and fluid-phase pinocytosis in quiescent fibroblasts by Ras proteins. Science (1986) 233(4768):1061-8. doi:10.1126/science.3090687

9. Veithen A, Cupers P, Baudhuin P, Courtoy PJ. v-Src induces constitutive macropinocytosis in rat fibroblasts. J Cell Sci (1996) 109(Pt 8):2005-12.

10. Commisso C, Davidson SM, Soydaner-Azeloglu RG, Parker SJ, Kamphorst JJ, Hackett S, et al. Macropinocytosis of protein is an amino acid supply route in Ras-transformed cells. Nature (2013) 497(7451):633-7. doi:10.1038/nature 12138

11. Kamphorst JJ, Nofal M, Commisso C, Hackett SR, Lu W, Grabocka E, et al. Human pancreatic cancer tumors are nutrient poor and tumor cells actively scavenge extracellular protein. Cancer Res (2015) 75(3):544-53. doi:10.1158/0008-5472.CAN-14-2211

12. Palm W, Park Y, Wright K, Pavlova NN, Tuveson DA, Thompson CB. The utilization of extracellular proteins as nutrients is suppressed by mTORC1. Cell (2015) 162(2):259-70. doi:10.1016/j.cell.2015.06.017

13. Swanson JA. Shaping cups into phagosomes and macropinosomes. Nat Rev Mol Cell Biol (2008) 9(8):639-49. doi:10.1038/nrm2447

14. West MA, Bretscher MS, Watts C. Distinct endocytotic pathways in epidermal growth factor-stimulated human carcinoma A431 cells. J Cell Biol (1989) 109(6 Pt 1):2731-9. doi:10.1083/jcb.109.6.2731

15. Commisso C, Flinn RJ, Bar-Sagi D. Determining the macropinocytic index of cells through a quantitative image-based assay. Nat Protoc (2014) 9(1):182-92. doi:10.1038/nprot.2014.004

16. Hoon JL, Wong WK, Koh CG. Functions and regulation of circular dorsal ruffles. Mol Cell Biol (2012) 32(21):4246-57. doi:10.1128/MCB.00551-12

17. Allison AC, Davies P, De Petris S. Role of contractile microfilaments in macrophage movement and endocytosis. Nat New Biol (1971) 232(31):153-5. doi:10.1038/newbio232153a0

18. Amyere M, Payrastre B, Krause U, Van Der Smissen P, Veithen A, Courtoy PJ.Constitutive macropinocytosis in oncogene-transformed fibroblasts depends on sequential permanent activation of phosphoinositide 3-kinase and phospholipase C. Mol Biol Cell (2000) 11(10):3453-67. doi:10.1091/mbc.11.10.3453

\section{ACKNOWLEDGMENTS}

Portions of the figure were created using images adapted from Servier Medical Art.

\section{FUNDING}

This work was supported by a Pancreatic Cancer Action Network-AACR Career Development Award (15-20-25COMM to CC) and a NIH-National Cancer Institute Research Project Grant (R01CA207189 to CC).

19. Egami Y, Taguchi T, Maekawa M, Arai H, Araki N. Small GTPases and phosphoinositides in the regulatory mechanisms of macropinosome formation and maturation. Front Physiol (2014) 5:374. doi:10.3389/fphys.2014.00374

20. Araki N, Johnson MT, Swanson JA. A role for phosphoinositide 3-kinase in the completion of macropinocytosis and phagocytosis by macrophages. J Cell Biol (1996) 135(5):1249-60. doi:10.1083/jcb.135.5.1249

21. Araki N, Egami Y, Watanabe Y, Hatae T. Phosphoinositide metabolism during membrane ruffling and macropinosome formation in EGF-stimulated A431 cells. Exp Cell Res (2007) 313(7):1496-507. doi:10.1016/j.yexcr.2007. 02.012

22. Miki H, Takenawa T. Regulation of actin dynamics by WASP family proteins. J Biochem (2003) 134(3):309-13. doi:10.1093/jb/mvg146

23. Di Paolo G, De Camilli P. Phosphoinositides in cell regulation and membrane dynamics. Nature (2006) 443(7112):651-7. doi:10.1038/nature05185

24. Veltman DM, Williams TD, Bloomfield G, Chen BC, Betzig E, Insall RH, et al. A plasma membrane template for macropinocytic cups. Elife (2016) 5:e20085 doi:10.7554/eLife.20085

25. Mellstrom K, Heldin $\mathrm{CH}$, Westermark B. Induction of circular membrane ruffling on human fibroblasts by platelet-derived growth factor. Exp Cell Res (1988) 177(2):347-59. doi:10.1016/0014-4827(88)90468-5

26. Ridley AJ, Paterson HF, Johnston CL, Diekmann D, Hall A. The small GTPbinding protein Rac regulates growth factor-induced membrane ruffling. Cell (1992) 70(3):401-10. doi:10.1016/0092-8674(92)90164-8

27. Nobes CD, Hawkins P, Stephens L, Hall A. Activation of the small GTPbinding proteins Rho and Rac by growth factor receptors. J Cell Sci (1995) 108(Pt 1):225-33.

28. Davidson SM, Jonas O, Keibler MA, Hou HW, Luengo A, Mayers JR, et al. Direct evidence for cancer-cell-autonomous extracellular protein catabolism in pancreatic tumors. Nat Med (2017) 23(2):235-41. doi:10.1038/nm.4256

29. Fujii M, Kawai K, Egami Y, Araki N. Dissecting the roles of Racl activation and deactivation in macropinocytosis using microscopic photo-manipulation. Sci Rep (2013) 3:2385. doi:10.1038/srep02385

30. Redelman-Sidi G, Iyer G, Solit DB, Glickman MS. Oncogenic activation of Pak1-dependent pathway of macropinocytosis determines BCG entry into bladder cancer cells. Cancer Res (2013) 73(3):1156-67. doi:10.1158/00085472.CAN-12-1882

31. Tajiri H, Uruno T, Shirai T, Takaya D, Matsunaga S, Setoyama D, et al. Targeting Ras-driven cancer cell survival and invasion through selective inhibition of DOCK1. Cell Rep (2017) 19(5):969-80. doi:10.1016/j.celrep.2017.04.016

32. Dharmawardhane S, Sanders LC, Martin SS, Daniels RH, Bokoch GM. Localization of p21-activated kinase 1 (PAK1) to pinocytic vesicles and cortical actin structures in stimulated cells. J Cell Biol (1997) 138(6):1265-78. doi:10.1083/jcb.138.6.1265

33. Dharmawardhane S, Schurmann A, Sells MA, Chernoff J, Schmid SL, Bokoch GM. Regulation of macropinocytosis by p21-activated kinase-1. Mol Biol Cell (2000) 11(10):3341-52. doi:10.1091/mbc.11.10.3341

34. Liberali P, Kakkonen E, Turacchio G, Valente C, Spaar A, Perinetti G, et al. The closure of Pak1-dependent macropinosomes requires the phosphorylation of CtBP1/BARS. EMBO J (2008) 27(7):970-81. doi:10.1038/emboj. 2008.59

35. Schnatwinkel C, Christoforidis S, Lindsay MR, Uttenweiler-Joseph S, Wilm M, Parton RG, et al. The Rab5 effector Rabankyrin-5 regulates and coordinates different endocytic mechanisms. PLoS Biol (2004) 2(9):E261. doi:10.1371/journal.pbio.0020261 
36. Porat-Shliom N, Kloog Y, Donaldson JG. A unique platform for H-Ras signaling involving clathrin-independent endocytosis. Mol Biol Cell (2008) 19(3):765-75. doi:10.1091/mbc.E07-08-0841

37. Lanzetti L, Palamidessi A, Areces L, Scita G, Di Fiore PP. Rab5 is a signalling GTPase involved in actin remodelling by receptor tyrosine kinases. Nature (2004) 429(6989):309-14. doi:10.1038/nature02542

38. Wennstrom S, Hawkins P, Cooke F, Hara K, Yonezawa K, Kasuga M, et al. Activation of phosphoinositide 3-kinase is required for PDGFstimulated membrane ruffling. Curr Biol (1994) 4(5):385-93. doi:10.1016/ S0960-9822(00)00087-7

39. Hawkins PT, Eguinoa A, Qiu RG, Stokoe D, Cooke FT, Walters R, et al. PDGF stimulates an increase in GTP-Rac via activation of phosphoinositide 3-kinase. Curr Biol (1995) 5(4):393-403. doi:10.1016/S0960-9822(95) 00080-7

40. Hewlett LJ, Prescott AR, Watts C. The coated pit and macropinocytic pathways serve distinct endosome populations. J Cell Biol (1994) 124(5):689-703. doi:10.1083/jcb.124.5.689

41. Racoosin EL, Swanson JA. Macropinosome maturation and fusion with tubular lysosomes in macrophages. J Cell Biol (1993) 121(5):1011-20. doi:10.1083/jcb.121.5.1011

42. Kerr MC, Lindsay MR, Luetterforst R, Hamilton N, Simpson F, Parton RG, et al. Visualisation of macropinosome maturation by the recruitment of sorting nexins. J Cell Sci (2006) 119(Pt 19):3967-80. doi:10.1242/jcs.03167

43. Dolat L, Spiliotis ET. Septins promote macropinosome maturation and traffic to the lysosome by facilitating membrane fusion. J Cell Biol (2016) 214(5):517-27. doi:10.1083/jcb.201603030

44. Koivusalo M, Welch C, Hayashi H, Scott CC, Kim M, Alexander T, et al. Amiloride inhibits macropinocytosis by lowering submembranous $\mathrm{pH}$ and preventing Racl and Cdc42 signaling. J Cell Biol (2010) 188(4):547-63. doi: $10.1083 /$ jcb. 200908086

45. Warburg O, Posener K, Negelein E. Über den Stoffwechsel der Carcinomzelle. Biochem Zeitschr (1924) 152:309-44.

46. Schornack PA, Gillies RJ. Contributions of cell metabolism and $\mathrm{H}+$ diffusion to the acidic $\mathrm{pH}$ of tumors. Neoplasia (2003) 5(2):135-45. doi:10.1016/ S1476-5586(03)80005-2

47. Estrella V, Chen T, Lloyd M, Wojtkowiak J, Cornnell HH, Ibrahim-Hashim A, et al. Acidity generated by the tumor microenvironment drives local invasion. Cancer Res (2013) 73(5):1524-35. doi:10.1158/0008-5472.CAN-12-2796

48. Fuster DG, Alexander RT. Traditional and emerging roles for the SLC9 $\mathrm{Na}+/ \mathrm{H}+$ exchangers. Pflugers Arch (2014) 466(1):61-76. doi:10.1007/ s00424-013-1408-8

49. Granja S, Tavares-Valente D, Queiros O, Baltazar F. Value of $\mathrm{pH}$ regulators in the diagnosis, prognosis and treatment of cancer. Semin Cancer Biol (2017) 43:17-34. doi:10.1016/j.semcancer.2016.12.003

50. Barretina J, Caponigro G, Stransky N, Venkatesan K, Margolin AA, Kim S, et al. The cancer cell line encyclopedia enables predictive modelling of anticancer drug sensitivity. Nature (2012) 483(7391):603-7. doi:10.1038/nature11003

51. Yoshimori T, Yamamoto A, Moriyama Y, Futai M, Tashiro Y. Bafilomycin A1, a specific inhibitor of vacuolar-type $\mathrm{H}(+)$-ATPase, inhibits acidification and protein degradation in lysosomes of cultured cells. J Biol Chem (1991) 266(26): 17707-12.

52. Kruth HS, Jones NL, Huang W, Zhao B, Ishii I, Chang J, et al. Macropinocytosis is the endocytic pathway that mediates macrophage foam cell formation with native low density lipoprotein. J Biol Chem (2005) 280(3):2352-60. doi:10.1074/jbc.M407167200

53. Kitazawa S, Nishizawa S, Nakagawa H, Funata M, Nishimura K, Soga T, et al. Cancer with low cathepsin D levels is susceptible to vacuolar $(\mathrm{H}+)$-ATPase inhibition. Cancer Sci (2017) 108(6):1185-93. doi:10.1111/cas.13240

54. Yumoto R, Suzuka S, Oda K, Nagai J, Takano M. Endocytic uptake of FITC-albumin by human alveolar epithelial cell line A549. Drug Metab Pharmacokinet (2012) 27(3):336-43. doi:10.2133/dmpk.DMPK-11-RG-127

55. Chung C, Mader CC, Schmitz JC, Atladottir J, Fitchev P, Cornwell ML, et al. The vacuolar-ATPase modulates matrix metalloproteinase isoforms in human pancreatic cancer. Lab Invest (2011) 91(5):732-43. doi:10.1038/ labinvest.2011.8
56. Cotter K, Capecci J, Sennoune S, Huss M, Maier M, Martinez-Zaguilan R, et al. Activity of plasma membrane V-ATPases is critical for the invasion of MDA-MB231 breast cancer cells. J Biol Chem (2015) 290(6):3680-92. doi:10.1074/jbc.M114.611210

57. Kimmelman AC. Metabolic dependencies in RAS-driven cancers. Clin Cancer Res (2015) 21(8):1828-34. doi:10.1158/1078-0432.CCR-14-2425

58. Lyssiotis CA, Son J, Cantley LC, Kimmelman AC. Pancreatic cancers rely on a novel glutamine metabolism pathway to maintain redox balance. Cell Cycle (2013) 12(13):1987-8. doi:10.4161/cc.25307

59. Son J, Lyssiotis CA, Ying H, Wang X, Hua S, Ligorio M, et al. Glutamine supports pancreatic cancer growth through a KRAS-regulated metabolic pathway. Nature (2013) 496(7443):101-5. doi:10.1038/nature12040

60. Ying H, Kimmelman AC, Lyssiotis CA, Hua S, Chu GC, FletcherSananikone E, et al. Oncogenic KRAS maintains pancreatic tumors through regulation of anabolic glucose metabolism. Cell (2012) 149(3):656-70. doi:10.1016/j.cell.2012.01.058

61. Kim MJ, Woo SJ, Yoon CH, Lee JS, An S, Choi YH, et al. Involvement of autophagy in oncogenic K-Ras-induced malignant cell transformation. J Biol Chem (2011) 286(15):12924-32. doi:10.1074/jbc.M110.138958

62. Lock R, Roy S, Kenific CM, Su JS, Salas E, Ronen SM, et al. Autophagy facilitates glycolysis during Ras-mediated oncogenic transformation. Mol Biol Cell (2011) 22(2):165-78. doi:10.1091/mbc.E10-06-0500

63. Perera RM, Stoykova S, Nicolay BN, Ross KN, Fitamant J, Boukhali M, et al. Transcriptional control of autophagy-lysosome function drives pancreatic cancer metabolism. Nature (2015) 524(7565):361-5. doi:10.1038/ nature 14587

64. Hosios AM, Hecht VC, Danai LV, Johnson MO, Rathmell JC, Steinhauser ML, et al. Amino acids rather than glucose account for the majority of cell mass in proliferating mammalian cells. Dev Cell (2016) 36(5):540-9. doi:10.1016/j. devcel.2016.02.012

65. Mayers JR, Torrence ME, Danai LV, Papagiannakopoulos T, Davidson SM, Bauer MR, et al. Tissue of origin dictates branched-chain amino acid metabolism in mutant KRAS-driven cancers. Science (2016) 353(6304):1161-5. doi:10.1126/science.aaf5171

66. Ma XM, Blenis J. Molecular mechanisms of mTOR-mediated translational control. Nat Rev Mol Cell Biol (2009) 10(5):307-18. doi:10.1038/nrm2672

67. Yoshida S, Pacitto R, Yao Y, Inoki K, Swanson JA. Growth factor signaling to mTORC1 by amino acid-laden macropinosomes. J Cell Biol (2015) 211(1):159-72. doi:10.1083/jcb.201504097

68. Olivares O, Mayers JR, Gouirand V, Torrence ME, Gicquel T, Borge L, et al. Collagen-derived proline promotes pancreatic ductal adenocarcinoma cell survival under nutrient limited conditions. Nat Commun (2017) 8:16031. doi:10.1038/ncomms16031

69. Qian Y, Wang X, Liu Y, Li Y, Colvin RA, Tong L, et al. Extracellular ATP is internalized by macropinocytosis and induces intracellular ATP increase and drug resistance in cancer cells. Cancer Lett (2014) 351(2):242-51. doi:10.1016/j.canlet.2014.06.008

70. Kamphorst JJ, Cross JR, Fan J, de Stanchina E, Mathew R, White EP, et al. Hypoxic and Ras-transformed cells support growth by scavenging unsaturated fatty acids from lysophospholipids. Proc Natl Acad Sci U S A (2013) 110(22):8882-7. doi:10.1073/pnas.1307237110

71. Michalopoulou E, Bulusu V, Kamphorst JJ. Metabolic scavenging by cancer cells: when the going gets tough, the tough keep eating. Br J Cancer (2016) 115(6):635-40. doi:10.1038/bjc.2016.256

Conflict of Interest Statement: The authors declare that the research was conducted in the absence of any commercial or financial relationships that could be construed as a potential conflict of interest.

Copyright (C) 2017 Recouvreux and Commisso. This is an open-access article distributed under the terms of the Creative Commons Attribution License (CC BY). The use, distribution or reproduction in other forums is permitted, provided the original author(s) or licensor are credited and that the original publication in this journal is cited, in accordance with accepted academic practice. No use, distribution or reproduction is permitted which does not comply with these terms. 